This document is the Accepted Manuscript version of a Published Work that appeared in final form as

\title{
"Mesoporous Semiconductors: A New Model to Assess Accessible Surface Area and Increased Photocatalytic Activity?"
}

in ACS Applied Energy Materials 20181 (11), 5787-5799, copyright $\odot$ American Chemical Society after peer review.

To access the final edited and published work see https://pubs.acs.org/doi/10.1021/acsaem.8b01123.

non-porous

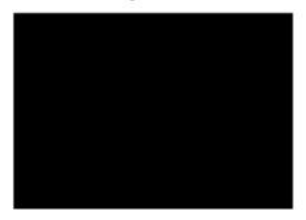

hard or soft templating

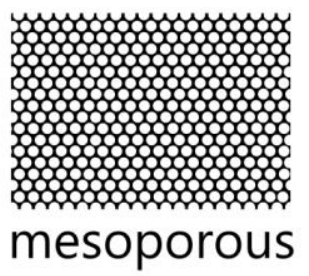

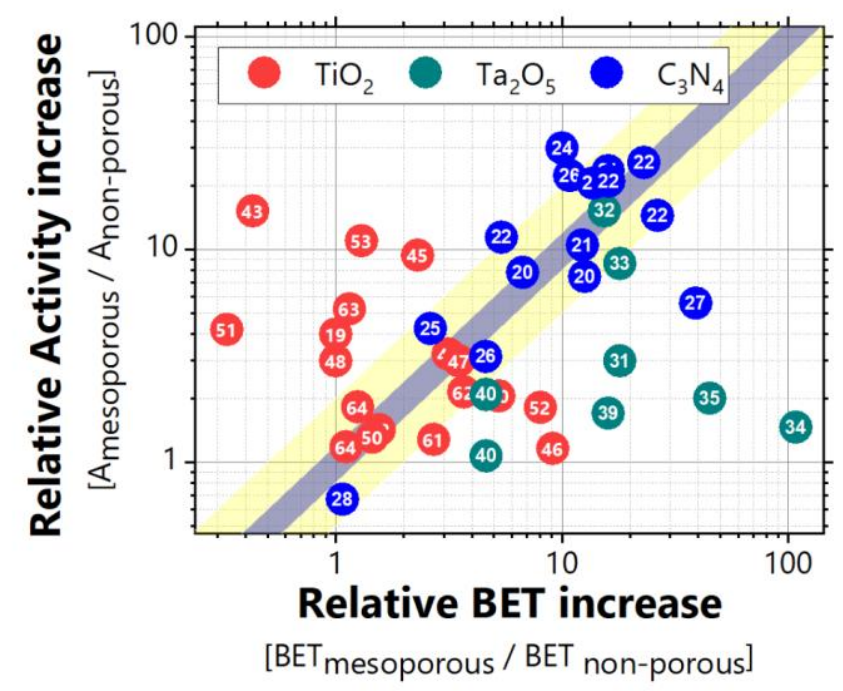




\title{
Mesoporous Semiconductors: A New Model to Assess Accessible Surface Area and Increased Photocatalytic Activity?
}

\author{
Alexey S. Cherevan ${ }^{*}$, Leonie Deilmann ${ }^{a}$, Tobias Wellerb, \\ Dominik Eder ${ }^{*}$, , and Roland Marschall ${ }^{*}, c$
}

\begin{abstract}
${ }^{a}$ A. S. Cherevan, L. Deilmann, D. Eder
Technische Universität Wien, Institut für Materialchemie, Getreidemarkt 9/BC/2, 1060 Vienna, Austria,

`Emails: dominik.eder@tuwien.ac.at ; alexey.cherevan@tuwien.ac.at

${ }^{\mathrm{b}} \mathrm{T}$. Weller, Justus-Liebig-University Giessen, Institute of Physical Chemistry, HeinrichBuff-Ring 17, 35392 Giessen, Germany

${ }^{\mathrm{c}} \mathrm{R}$. Marschall, University of Bayreuth, Chair of Physical Chemistry III, Universitätsstr. 30, 95447 Bayreuth, Germany

*Email: roland.marschall@uni-bayreuth.de
\end{abstract}

Keywords: mesoporous materials; porosity; templating; photocatalysis; hydrogen generation; $\mathrm{CO}_{2}$ reduction

Abstract: Mesoporous photocatalysts have gained tremendous attention in the last decade by demonstrating that increased surface area and porosity can strongly improve their performance. In fact, all reports on mesoporous semiconductors corroborate this scenario. But is it possible to quantify and compare the reported advantages of the mesopores and the increased surface area between different works? In this contribution, we present a model that can evaluate the improvements in photocatalytic activity achieved by the introduction of mesoporosity independent on synthetic or test conditions. We exemplify this methodology focusing on photocatalytic hydrogen/oxygen evolution with sacrificial reagents, but also include examples of $\mathrm{CO}_{2}$ reduction and electrocatalysis. By correlating the relative increase in surface area to the relative increase in activity - in comparison to non-porous counterparts - we show that the origin of mesoporosity can have a pronounced influence on the activity enhancement, and that different semiconductor materials behave quite differently. Our model can serve as a starting point for the community to extract and compare key information on mesoporous photocatalysts, to put results into context of existing data, and to compare the performances of various catalytic systems much better. 


\section{Introduction}

The search for sustainable and renewable energy sources to substitute current fossil fuels and to reduce the emission of greenhouse gases in our atmosphere for a cleaner environment constitutes one of the key fundamental challenges of our modern society. ${ }^{1}$

The ability to harness sunlight for the conversion of abundant materials, such as water and $\mathrm{CO}_{2}$, into useful chemicals and fuels has the potential to revolutionize the field of green chemistry and pave the way towards a more sustainable way of life. In fact, the splitting of water into its constituent elements, oxygen and hydrogen, remains the key requirement to realizing the successful use of hydrogen as a clean energy source and was recently identified by the European Science Foundation as one of the world's emerging key research fields. ${ }^{2}$ Light-driven conversion of $\mathrm{CO}_{2}$ into chemical commodities for industrial production of fine chemicals would equally benefit environment and economy. ${ }^{3}$

Another global challenge is to provide clean water, both from accessing natural water resources and from recycling and purifying wastewater. Industrial and agricultural wastes produce a variety of aromatic compounds, while xenobiotic substances (i.e. antibiotics, drugs, toxins, pesticides) are becoming an increasingly large problem, since they are often resistant towards degradation with currently applied purification techniques. ${ }^{4}$

Semiconductor photocatalysis and photoelectrochemistry can offer very promising pathways towards clean energy and water, as highlighted by the continuously increasing numbers of publications and patents. Yet, despite the tremendous developments over the last decade, still more efficient, stable, recyclable, and recoverable photocatalysts and photoelectrodes are required.

A plethora of strategies have recently been developed to design new types of photoactive materials, which typically address one or two of the many performance-limiting characteristics, such as light absorption, degree of crystallinity, surface faceting, accessibility of catalytic sites, lifetime of charge carriers, charge transport and charge extraction. These include, for example, heteroatom doping, ${ }^{5}$ defect and crystal engineering, ${ }^{6}$ and surface modification of the semiconducting catalyst, ${ }^{7}$ as well as the 
coupling and interface engineering of the semiconductor with another catalyst (e.g. Zscheme),${ }^{8}$ co-catalyst, ${ }^{9}$ photosensitizer ${ }^{10}$ or charge acceptor. ${ }^{11}$ Other popular strategies include the down-scaling to the nanometer regime and the structuring into $1 \mathrm{D}$ and $2 \mathrm{D}$ nanomaterials to increase surface area and charge transport characteristics. ${ }^{12}$

Recently, a review addressing the performance limiting factors in photocatalytic and photosynthetic processes indicated that surface area is the sole limiting parameter for photocatalytic devices (e.g. $\mathrm{H}_{2}$ evolution with sacrificial reagent from particle suspensions) when the reaction is carried out upon conditions of sufficient charge carrier generation, long carrier lifetimes, and low overpotentials. ${ }^{13}$ Therefore, an increase in surface area is considered beneficial to enhance photocatalytic activity. However, this analysis assumes that all surface area is accessible.

Prominent strategies to enhance the surface area of semiconductor materials are ball milling of bulk materials, ${ }^{14}$ synthesis of nanoparticles, ${ }^{15}$ and nano/mesostructuring of bulk materials to produce porous catalysts, typically using soft or hard templates. ${ }^{16} \mathrm{~A}$ new research area that focuses on the implementation and engineering of mesopores to facilitate access to a large interior active surface area has therefore gained tremendous popularity. ${ }^{17}$ Porous photocatalysts derived in these ways often exhibit a larger specific surface area, which is typically measured by inert gas physisorption measurements (e.g. using $\mathrm{N}_{2}$ or $\mathrm{Ar}$ ) and calculated by e.g. non-local DFT or Brunauer-Emmet-Teller (BET) method using specific isotherms. However, for systems with micropores or small mesopores, the measurement of surface area and porosity characteristics (pore size distribution etc.) becomes a challenge. As such, poorly measured isotherms or wrongly applied theoretical models can result in highly inaccurate values. This problem is unfortunately rarely discussed in application-driven papers, thus leaving the readers with just one option to trust the reported values, while still introducing one more factor of uncertainty. Yet, the main question remains whether the entire measured surface area is fully accessible to reactant molecules during the photocatalytic process. Does the existing literature hold an answer?

To address this issue, here we present a methodology that allows evaluating the accessibility of the surface area in correlation with the photocatalytic activity for a range 
of process parameters. Our approach further stimulates a database that potentially enables a comprehensive comparison of all (photo)catalysts and (photo)electrodes reported in literature independent of the many existing process conditions.

Key to this methodology is the extraction of the unit-free terms "relative activity increase" and "relative BET increase", which describe the factor of activity increase (from evolution rates, photonic efficiencies, IPCE or photocurrent values) and the factor of specific surface area (from BET model) increase between the mesoporous photocatalyst and a non-porous reference (Scheme 1). This methodology allows to eradicate or at least consider many internal parameters (such as reaction conditions, material of choice, catalyst amounts and synthetic protocols) of each individual report and, therefore, compare the data extracted from a large number of articles. We would have preferred to extract the increase of activity from quantum efficiencies, but still very few reports on mesoporous photocatalysts provided quantum efficiencies, so in most of the cases we calculated the activity increase from evolution rates.
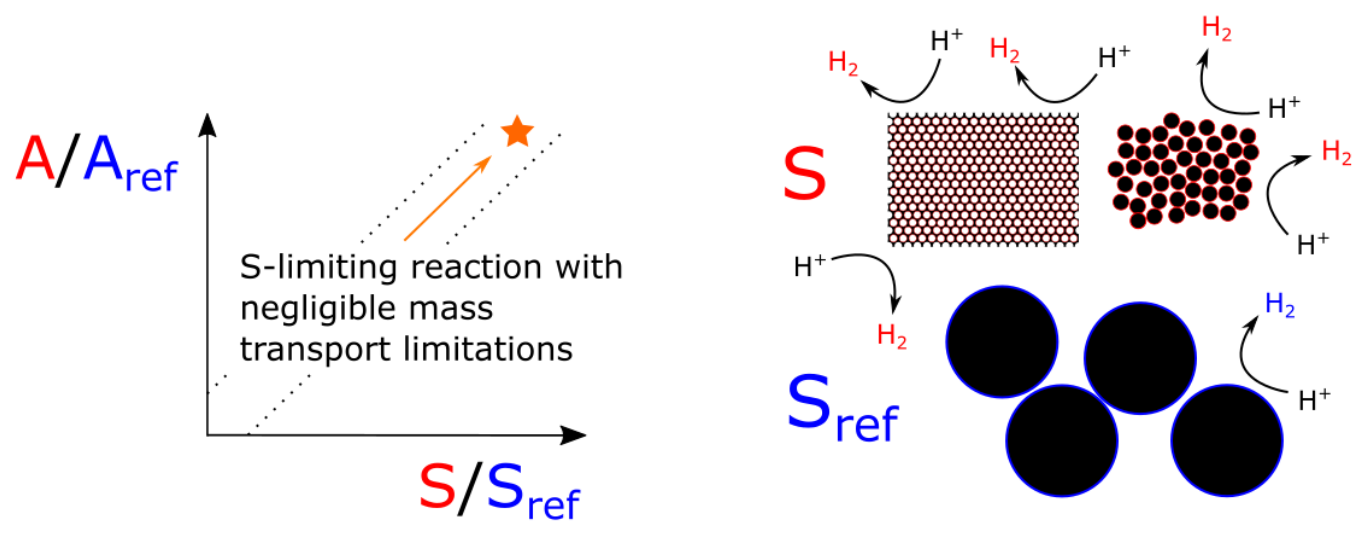

Scheme 1 Illustration of the methodology used to evaluate the bulk of the literature data. $S$ and $S_{\text {ref }}$ correspond to the surface area of the mesoporous catalyst (e.g. ordered structures or non-ordered agglomerates, in red) and the non-porous reference (e.g. typically particle aggregates, in blue) reported alongside, respectively. $A$ and $A_{\text {ref }}$ correspond to activity of the mesoporous catalyst and of the non-porous reference reported alongside, respectively.

We will exemplify this methodology for photocatalytic hydrogen production with sacrificial reagents, which is a photocatalytic downhill reaction in which the surface area has been proposed to determine the photocatalytic activity, as recently highlighted by Osterloh. ${ }^{13}$ Accordingly, full accessibility of the surface area constitutes the rate limiting factor, and all other parameters are considered secondary under the given conditions. However, the 
here presented new methodology can be used to analyze many porous systems, including electrocatalysts, (photo)electrodes, and hierarchical porous photocatalysts ${ }^{18}$ in view of a wide range of reactions. Thus, we also looked at some oxygen evolution and $\mathrm{CO}_{2}$ reduction reactions, yet we excluded dye degradation reactions due to the large variety of organic compounds and additional experimental parameters used in these works. We also excluded metal-organic frameworks and covalent-organic frameworks from our analysis due to the limited number of examples for photocatalysis or photolectrochemistry reported so far. ${ }^{19-20}$

\section{Database}

We have analyzed roughly 200 papers (full list of references is available in the $\mathrm{SI}$ ) in which mesoporous materials have been synthesized and evaluated regarding their activity in various photocatalytic applications. Driven by the idea to identify current trends of the state-of-the-art research and be able to compare results obtained in different works, we were especially interested in reports in which non-porous references had been investigated in comparison with the mesoporous catalysts. It should be noted that such references ideally constitute non-porous counterparts of the mesoporous materials being fully characterized regarding surface area, crystallinity and photocatalytic performance.

We then chose about 50 examples in which the effect of mesoporosity on activity was analyzed by comparing the performance (evolution rates) of the mesoporous sample with such a reference material. These papers - presented in Table 1 - laid the foundation for our data analysis that is presented at the beginning of the next chapter.

In particular, we have categorized the results regarding important criteria, including the type of semiconductor, origin of porosity, and size/structure of mesopores; we analyzed how surface area increase and photocatalytic activity are connected for porous materials and identified other factors that may contribute to the photocatalytic activity of a mesoporous network. More specifically, we have compared the activity changes (enhancement in $\mathrm{H}_{2}$ evolution, $\mathrm{O}_{2}$ evolution or $\mathrm{CO}_{2}$ reduction rates with sacrificial reagents) with the surface area enlargement upon introduction of mesopores. This 
allowed us to extract the aforementioned unit-free terms "relative activity increase" and "relative BET increase", which are discussed in the next chapter.

\subsection{Observations}

Type of material: As expected, the most frequently studied mesoporous photocatalysts have been $\mathrm{TiO}_{2}$ or $\mathrm{TiO}_{2}$-based systems, as highlighted by Table 1. Other materials of interest for photocatalytic applications included carbon nitrides, which were recently reviewed regarding synthesis and applications ${ }^{21}$, and tantalum oxide-based systems. Only a minor part of the reports aimed at introducing functional mesoporosity into less common materials such as sulfides, vanadates, nitrides as well as $\mathrm{Nb}_{2} \mathrm{O}_{5}$ and $\mathrm{Ga}_{2} \mathrm{O}_{3}-$ based catalysts.

Synthesis route: Most of the mesoporous photo(electro)catalysts have been prepared by means of hard-templating (with $\mathrm{SiO}_{2}$ nanoparticles (NP), mesoporous $\mathrm{SiO}_{2}$, polystyrene (PS), polymethylmethacrylate (PMMA), cellulose, or biological systems such as bacteria or butterfly wings as templates) or soft-templating (with block copolymers or surfactants) routes (Table 1). A number of articles obtained mesoporous structures without the use of any templates, for example, via modified solvothermal or sol-gel processes. However, only structures with unordered pore geometry have been obtained in this way, most often nanoparticle assemblies with interparticular porosity.

Materials characterization: Most catalysts were generally characterized by X-ray powder diffraction (XRPD, to identify phase), transmission and scanning electron microscopy (TEM and SEM, to reveal morphology, pore dimensions and order) and physisorption (to estimate surface area and pore size distribution). Some studies involved X-ray photoelectron spectroscopy (XPS, to confirm composition and purity) and diffuse reflection spectroscopy (DRS, to evaluate light absorption properties). The latter is quite an important point that makes it possible to analyze whether mesoporosity does affect light absorption of mesoporous photocatalysts. Very few have combined all techniques in the same report. 
Table 1 Summary of the materials characteristics from papers that contributed to our statistical analysis

\begin{tabular}{|c|c|c|c|c|c|c|c|c|}
\hline Material & Order & $\begin{array}{l}\text { Pore } \\
\text { architecture }\end{array}$ & Synthesis method [template] & Pore size [nm] & $\begin{array}{l}\text { Pore size } \\
\text { distributions } \\
{[\mathrm{nm}]}\end{array}$ & $\begin{array}{l}\text { Surface area } \\
\text { BET } \\
{\left[\mathrm{m}^{2} / \mathrm{g}\right]}\end{array}$ & Application & Ref \\
\hline $\mathrm{C}_{3} \mathrm{~N}_{4}$ & NO & wormhole-like & hard $\left[\mathrm{SiO}_{2} \mathrm{NPs}\right]$ & 8.3 & $5-15$ & 67 & PC HER & 22 \\
\hline $\mathrm{C}_{3} \mathrm{~N}_{4}$ & NO & - & hard [in situ $\left.\mathrm{SiO}_{2} \mathrm{NPs}\right]$ & $4-5$ & broad & $123,137,160$ & PC HER & 23 \\
\hline $\mathrm{C}_{3} \mathrm{~N}_{4}$ & - & - & $\begin{array}{l}\text { template-free, modified sol- } \\
\text { gel }\end{array}$ & 3.5 & - & $\begin{array}{l}54,161,230 \\
264,273\end{array}$ & PC HER & 24 \\
\hline $\mathrm{C}_{3} \mathrm{~N}_{4}$ & $\mathrm{O}$ & cylindrical & hard [SBA-15] & 5.3 & $2-12$ & 239 & PC HER & 25 \\
\hline $\mathrm{C}_{3} \mathrm{~N}_{4}$ & NO & - & template-free, hydrothermal & 9 & $2-20$ & 130 & PC HER & 26 \\
\hline $\mathrm{C}_{3} \mathrm{~N}_{4}$ & NO & interparticular & hard [in situ $\left.\mathrm{SiO}_{2} \mathrm{NPs}\right]$ & 3.7 & - & 170 & PC HER & 27 \\
\hline $\mathrm{C}_{3} \mathrm{~N}_{4}$ & NO & spherical & hard $\left[\mathrm{SiO}_{2} \mathrm{NPs}\right]$ & 7 & $10-20$ & 131 & PC HER & 28 \\
\hline $\mathrm{C}_{3} \mathrm{~N}_{4}$ & NO & - & hard $\left[\mathrm{SiO}_{2} \mathrm{NPs}\right]$ & - & - & 216 & PC HER & 29 \\
\hline $\mathrm{CN}_{\mathrm{x}}$ & NO & interparticular & hard [in situ $\left.\mathrm{SiO}_{2}\right]$ & 3.6 & $2-13$ & 167 & PC HER & 30 \\
\hline $\mathrm{S}-\mathrm{C}_{3} \mathrm{~N}_{4}$ & NO & spherical & hard $\left[\mathrm{SiO}_{2} \mathrm{NPs}\right]$ & 7 & $10-20$ & 128 & PC HER & 28 \\
\hline $\mathrm{Nb}_{2} \mathrm{O}_{5}$ & NO & interparticular & soft [P123] & 7.7 & $2-30$ & 89,108 & PC HER & 31 \\
\hline $\mathrm{Nb}_{2} \mathrm{O}_{5}$ & NO & wormhole-like & soft [P123] & 6 & $2-25$ & 96 & PC HER & 32 \\
\hline $\mathrm{Ta}_{2} \mathrm{O}_{5}$ & NO & wormhole-like & soft [P123] & 12.1 & 3-15 & 41 & PC HER \& OER & 33 \\
\hline $\mathrm{Ta}_{2} \mathrm{O}_{5}$ & 0 & gyroidal & soft [ISO polymer] & 28 & $15-45$ & 34 & PC HER & 34 \\
\hline $\mathrm{Ta}_{2} \mathrm{O}_{5}$ & 0 & cylindrical & soft [P123] & 3.7 & - & 109 & PC HER \& OER & 35 \\
\hline $\mathrm{Ta}_{2} \mathrm{O}_{5}$ & 0 & cylindrical & soft [P123] & 3 & 3 & 108 & PC HER & 36 \\
\hline $\mathrm{Ta}_{2} \mathrm{O}_{5}$ & NO & - & soft [P123] & 2.3 & 2.3 & 92 & PC HER & 37 \\
\hline $\mathrm{Ta}_{2} \mathrm{O}_{5}$ & NO & interparticular & soft [P123] & 5.4 & $2-14$ & 117 & PC HER & 38 \\
\hline $\mathrm{NaTaO}_{3}$ & NO & interparticular & hard $\left[\mathrm{SiO}_{2} \mathrm{NPs}\right]$ & 20,50 & - & 46,26 & PC HER \& OER & 39 \\
\hline $\mathrm{NaTaO}_{\mathrm{x}}$ & NO & interparticular & template-free, hydrothermal & - & $10-25$ & - & PC HER \& OER & 40 \\
\hline $\mathrm{CsTaWO}_{6}$ & NO & - & soft [P123] & 10 & $5-20$ & 78 & PC HER & 41 \\
\hline $\mathrm{N}-\mathrm{Ta}_{2} \mathrm{O}_{5}$ & NO & interparticular & soft [SDA] & 6.8 & - & 86 & PC HER \& $\mathrm{CO}_{2}$ & 42 \\
\hline $\mathrm{Ta}_{3} \mathrm{~N}_{5}$ & 0 & cylindrical & soft [P123], ammonolysis & 3.7 & $2-8$ & 114 & PC HER & 43 \\
\hline $\mathrm{Ta}_{3} \mathrm{~N}_{5}$ & NO & interparticular & hard $\left[\mathrm{C}_{3} \mathrm{~N}_{4}\right]$, ammonolysis & $7,12,24$ & - & $61,45,40$ & PC HER & 44 \\
\hline $\mathrm{TiO}_{2}$ & NO & interparticular & template-free & 3.9 & $2-4$ & 70 & PC HER & 45 \\
\hline $\mathrm{TiO}_{2}$ & 0 & cylindrical & soft [P123] & 4.6 & $2-5$ & 169 & PC HER & 46 \\
\hline $\mathrm{TiO}_{2}$ & NO & wormhole-like & soft [P123] & 6.2 & $3-7$ & 173 & PC HER & 46 \\
\hline $\mathrm{TiO}_{2}$ & NO & interparticular & template-free, electrospinning & 7 & $1-15$ & 96 & PC HER & 47 \\
\hline $\mathrm{TiO}_{2}$ & NO & - & soft [CTAB], hydrothermal & 2.1 & - & 438 & PC HER & 48 \\
\hline $\mathrm{TiO}_{2}$ & $\mathrm{O}$ & cylindrical & soft [F127] & $7.2,6.8,6.5$ & $2-10$ & $267,209,175$ & PC HER & 49 \\
\hline $\mathrm{TiO}_{2}$ & NO & - & soft [F127] & 8.5 & $2-15$ & 54 & PC HER & 50 \\
\hline $\mathrm{TiO}_{2}$ & NO & wormhole-like & soft [TEA] & 4.5 & $3-5$ & 525 & PC HER & 51 \\
\hline $\mathrm{S}, \mathrm{N}-\mathrm{TiO}_{2}$ & NO & interparticular & $\begin{array}{l}\text { template-free, precipitation \& } \\
\text { deposition }\end{array}$ & $8.1,10.7,33$ & $\begin{array}{l}2-10,4-13,5- \\
40\end{array}$ & $114,80,21$ & PC HER & 52 \\
\hline $\mathrm{SrTiO}_{3}$ & NO & interparticular & soft [SDA] & 5 & $2-9$ & 10 & PC HER & 53 \\
\hline $\mathrm{SrTiO}_{3}$ & NO & interparticular & soft [SDA], hydrothermal & $2.5 \& 30,2.5 \& 90$ & $2-100$ & 101,65 & PC OER & 54 \\
\hline $\mathrm{SrTiO}_{3}$ & $\mathrm{O}$ & spherical & hard [PMMAs \& PSs] & 150 & - & 23 & PC HER & 55 \\
\hline CdS & NO & - & template-free, ultrasonication & 5.4 & $0.5-9$ & 95 & PC HER & 56 \\
\hline $\mathrm{InVO}_{4}$ & NO & wormhole-like & soft [CTAB] & - & - & 66,45 & PC HER & 51 \\
\hline $\mathrm{Mn}_{2} \mathrm{O}_{3}$ & NO & - & soft [P123] & 9.6 & $5-15$ & 120 & PC OER & 57 \\
\hline $\mathrm{WO}_{3}$ & $\mathrm{O}$ & spherical & soft & 21 & $10-50$ & 70 & PC OER & 58 \\
\hline $\mathrm{ZnS}-\mathrm{ZnO}$ & NO & - & template-free, [hydrothermal & 5.3 & $2-15$ & 35 & PC HER & 59 \\
\hline $\mathrm{Bi}_{2} \mathrm{WO}_{6}$ & 0 & spherical & hard [PSs] & 405 & - & - & PEC HER & 60 \\
\hline $\mathrm{BiVO}_{4}$ & $\mathrm{O}$ & dual porosity & hard [PSs] & $\begin{array}{l}155 \& 105,170 \& \\
85,180 \& 35\end{array}$ & - & - & PEC HER \& OER & 61 \\
\hline $\mathrm{Fe}_{2} \mathrm{O}_{3}$ & NO & - & hard $\left[\mathrm{SiO}_{2} \mathrm{NPs}\right]$ & 10 & $10-30$ & 18 & PEC OER & 62 \\
\hline $\mathrm{TiO}_{2}$ & NO & interparticular & soft [P123] & 12 & $5-12$ & 103 & PEC HER & 63 \\
\hline $\mathrm{TiO}_{2}$ & $\mathrm{O}$ & spherical & hard [PSs] & 290 & - & 34 & PEC HER & 64 \\
\hline $\mathrm{TiO}_{2}$ & NO & wormhole-like & soft [P123] & 7 & $2-10$ & 125 & PEC HER & 64 \\
\hline $\mathrm{TiO}_{2}$ & NO & wormhole-like & soft [P123] & 5.1 & - & 197 & PEC HER & 65 \\
\hline $\mathrm{TiO}_{2}$ & $\mathrm{O}$ & cylindrical & soft [P123] \& hard [PSs] & $5.3 \& 255$ & - & 228 & PEC HER & 65 \\
\hline $\mathrm{TiO}_{2}$ & NO & interparticular & soft [SDA] & 3 & $3-5$ & $94,105,117$ & PEC OER & 66 \\
\hline $\mathrm{TiO}_{2}$ & NO & interparticular & $\begin{array}{l}\text { template-free, supercritical } \\
\text { drying }\end{array}$ & 40 & $5-100$ & 114 & PEC OER & 67 \\
\hline $\mathrm{Ga}_{2} \mathrm{O}_{3}$ & NO & - & soft & 4 & $3-30$ & 42 & $\mathrm{CO}_{2}$ & 68 \\
\hline $\mathrm{TiO}_{2}$ & NO & - & template-free & $2.3,12.4$ & $2-50$ & 50 & $\mathrm{CO}_{2}$ & 69 \\
\hline $\mathrm{ZnGa}_{2} \mathrm{O}_{4}$ & NO & wormhole-like & template-free, hydrothermal & 3.5 & $3-4$ & 110 & $\mathrm{CO}_{2}$ & 70 \\
\hline
\end{tabular}

O - ordered; NO - non-ordered; SDA - structure directing agent; NP - nanoparticle; PS - polystyrene; PMMA - polymethylmethacrylate; PC photocatalytic; $\quad$ PEC - photoelectrocatalytic; $\mathrm{HER}$-hydrogen evolution reaction; $\mathrm{OER}-\mathrm{oxygen}$ evolution reaction; $\mathrm{CO}_{2}-$ photo(electro)catalytic $\mathrm{CO}_{2}$ reduction 
Type of application: In contrast to the field of photocatalysis, the measurement of specific surface areas and their correlation with performance has often been neglected in the photoelectrochemistry community. ${ }^{71}$ This is likely due to the often small amounts of material used and their device-based synthesis, which render surface area measurements challenging. Consequently, only a small number of PEC-related papers could be evaluated in this article.

\section{Analysis and Discussion}

The accessibility of the internal surface area is typically defined by the minimum width of the pore, which - often near the pore opening - can also be blocked during diffusion processes. To minimize kinetic restriction, research targets pore diameters in the mesopore regime $(2-50 \mathrm{~nm})$ with a focus on pores larger than $10 \mathrm{~nm}$. Pore size distributions can have additional non-linear effects on educt and product diffusion. Pore length and - in conjunction with the pore width - aspect ratio are also critical parameters. The diffusion characteristics are also affected by the surface chemistry of the pore walls through adsorptive interactions with the reactant molecules. Moreover, the pore architecture defines the overall pore connectivity. For example, diffusion through 1D pores is more dependent on aspect ratio and pore blocking than diffusion through 3Dinterconnected pore architectures, such as in gyroidal structures. ${ }^{72}$ The pore architecture and ordering can further affect the performance of photocatalysts by enhancing light propagation and enabling photonic characteristics. ${ }^{69}$ Finally, the crystallinity and dimensions of the pore walls can affect the diffusion of photogenerated charge carriers, which can also be considered as educts, from the interior to the surface of the catalyst/electrode. ${ }^{2}$

\subsection{Analyzing the Database}

We have analyzed literature data according to enhancements in photocatalytic activity due to an increase in surface area by establishing mesoporosity in the final product. Figure 1 shows the analyzed database and the dependence of activity increase factor on surface area enlargement factor for different photocatalytic applications. 

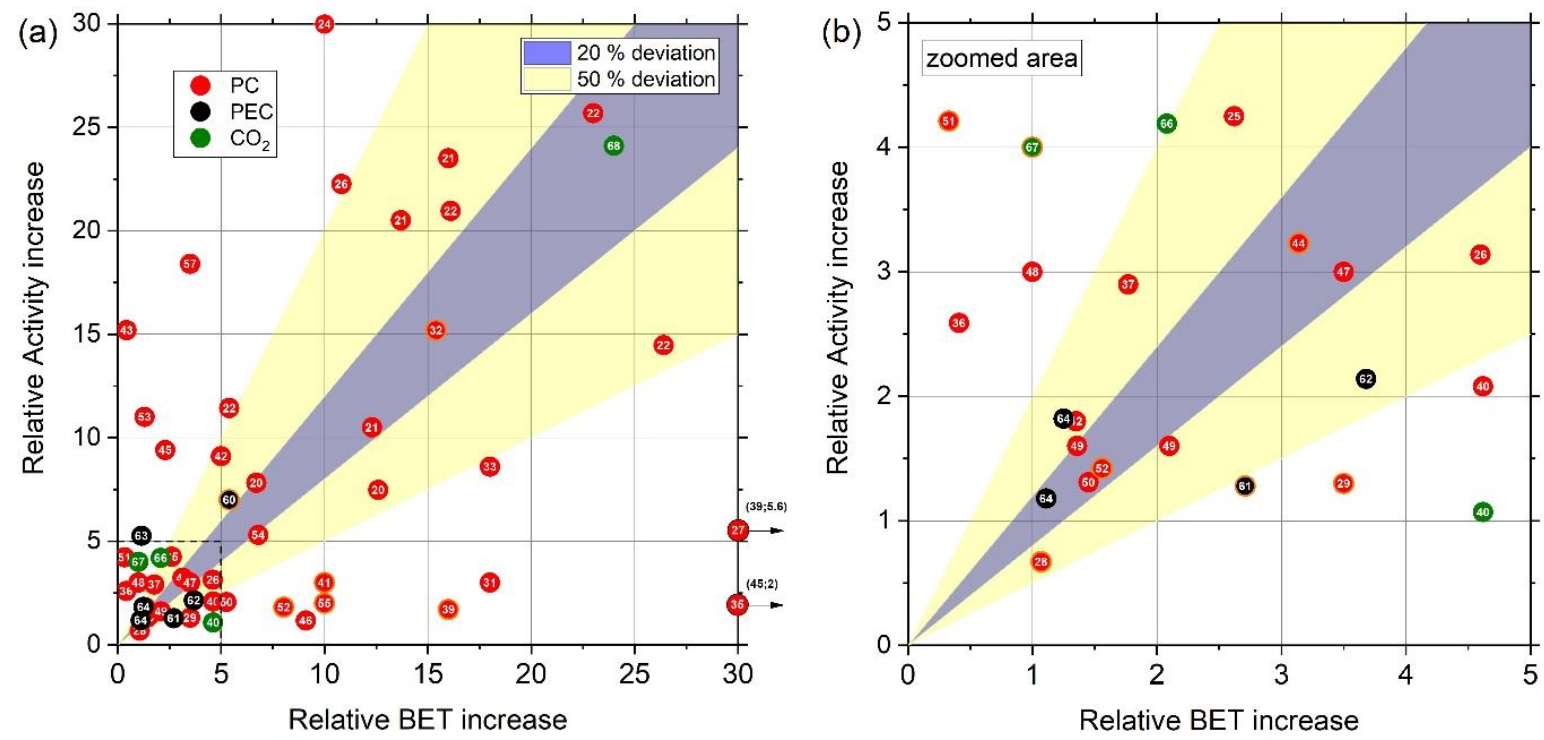

Figure 1 Correlation of increase in photocatalytic or photoelectrochemical activity with increase in BET surface area, always related to a non-porous reference: (a) all analyzed papers; (b) magnification of the bottom left area of the left graph. Different colors represent different application. Colored areas represent deviation from the linear surface-activity correlation. Numbers inside the data points correspond to the reference number of the manuscript in the main reference list. References that are discussed in detail are highlighted by a golden circle.

Each of the colored dots in Figure 1 represent one example (mesoporous photocatalyst and a corresponding non-porous reference) from our literature database: red dots indicate photocatalytic (PC) hydrogen evolution reaction (HER) or oxygen evolution reaction (OER) results, black dots correspond to photoelectrochemical (PEC) studies, and green dots depict photocatalytic $\mathrm{CO}_{2}$ reduction results. The blue and yellow shaded areas highlight $\mathrm{a} \pm 20 \%$ and $\mathrm{a} \pm 50 \%$ deviation range from the projected linear increase of activity with surface area.

It can be seen immediately from Figure 1 that only very few studies give a close to linear correlation of activity increase with surface area enlargement due to our proposed model. The data for activity increase are highly scattered, even in the region of small values of surface area increase (Figure 1b). Fundamentally speaking, an increase in surface area should lead to a linear increase in the number of catalytic active sites on the surface and therefore photocatalytic activity. Yet, the extracted data in Figure 1 suggest that this linear correlation is not governed solely by the surface area, in contrast to the considerations by 
Osterloh; hence, we have to consider other contributing factors, i.e. kinetic limitations that can affect the photocatalytic performance of porous photocatalysts.

We can envision several potential reasons for this large deviation from a linear correlation. The simplest explanation involves mass transport limitation of reactants, products or electrolytes, when small pores are present. An increase in surface area with respect to the active sites is only beneficial if these are fully accessible, while recombination centers don't need accessibility and thus may at one point become the dominant contribution, leading to non-linear increase in activity with surface area. A few years ago, Li et al. have investigated the degradation of methylene blue over ordered mesoporous $\mathrm{WO}_{3}$ photocatalysts with narrow pore size distributions that ranged from 8 to $25 \mathrm{~nm}^{73}$, and observed considerable kinetic limitations by reactant diffusion even in pores with diameters up 10-12 nm. If we consider that many literature reports have described materials with a broad rather than narrow pore size distribution, i.e. including micropores, it is plausible to expect diffusion limitations and thus a deviation from linear correlation with activity. Note that this becomes particularly problematic when using nanoparticle aggregates, which are often denominated as "mesoporous catalysts".

Another explanation involves the crystallinity of the newly designed mesoporous catalysts, which can differ from the reference material, thus introducing yet another contribution to the non-linear performance.

Therefore, we have narrowed down our dataset and only considered those reports that have fully characterized and discussed the catalysts' pore sizes and pore size distributions. Figure 2a shows, now on a double-logarithmic scale, the activity vs surface area trend for different average pore sizes. Interestingly, as can be seen from the distribution profile, the non-linear correlation between activity increase factor and surface area enlargement factor that we observed before (in Figure 1) does not originate from different pore dimensions. We have chosen $5 \mathrm{~nm}$ pores as a threshold for our data analysis, because it is the typical pore size one can achieve synthetically using the commercial block-copolymer Pluronic P123 as a porogen. ${ }^{74}$ Still, Figure 2a illustrates nonlinear behavior and large scattering of data points independent on the average pore size. 
Some literature reported a linear activity increase with surface area also for pores smaller than $5 \mathrm{~nm}$ (e.g. data points in the blue area). This is in contrast to some more recent literature reports, which indicated that pore blocking in photocatalytic water splitting/hydrogen production could only be avoided when sufficiently large pores $(>10 \mathrm{~nm})$ are present. ${ }^{34}$ Our data analysis clearly shows that neither of the two statements can in fact be generalized, independent of the three chosen applications.

Another reason for the highly-scattered results in Figure 1 might be that neither pore geometry (ordered, non-ordered, broad or narrow pore size distribution) nor pore wall thickness have been considered so far, which are indeed crucial characteristics that affect the photo(electro)catalytic performance. Importantly, the "optimum" pore wall thickness, i.e. in view of charge transport to the solid-liquid interface, will vary for different mesoporous materials due to varying diffusion length of charge carriers in each material. Thus, differences in material nature need to be considered. Yet, a linear relation of activity with surface area enlargement is still expected with every material. The question remains as to which of the structural parameters limit the performance in each case.
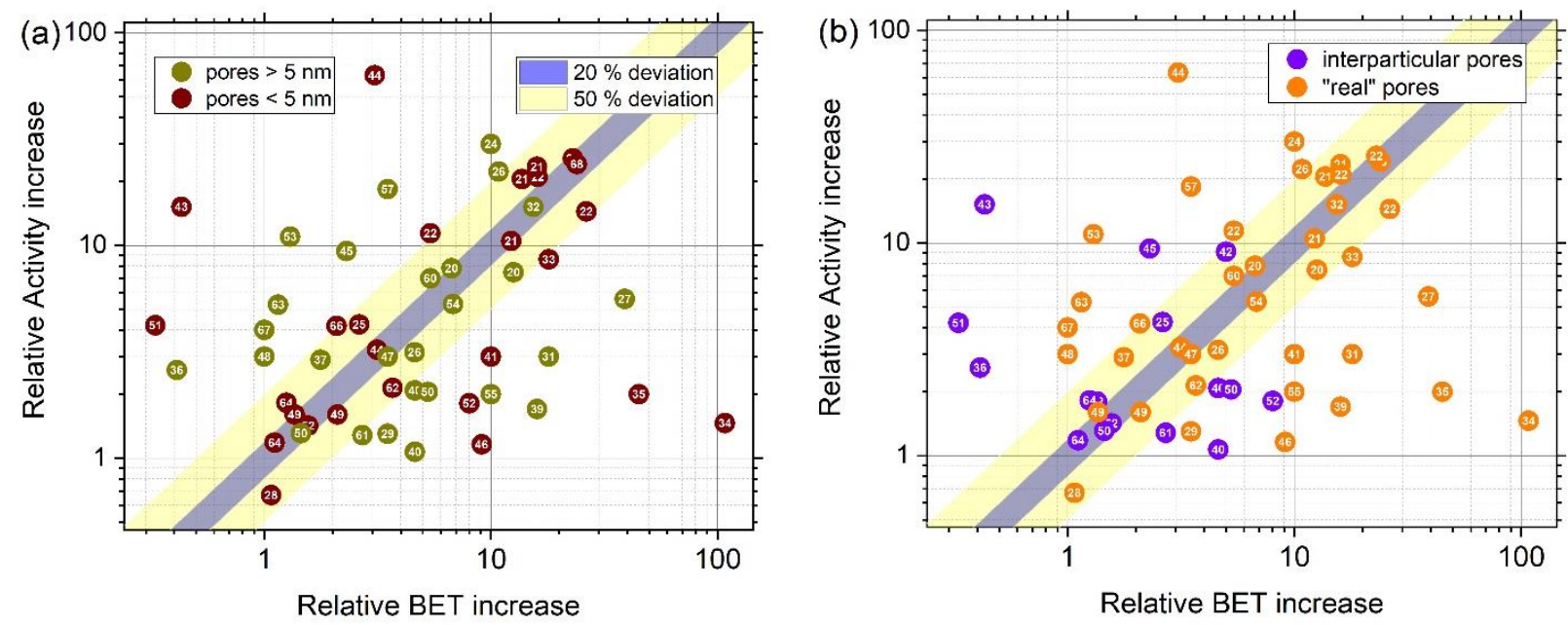

Figure 2 Correlation of increase in photocatalytic or photoelectrochemical activity with increase in BET surface area, both related to a non-porous reference as a function of (a) pore size; (b) pore origin. Colored areas represent deviation from the linear surface-activity correlation. Numbers inside the data points correspond to the reference number of the manuscript in the main reference list. 
We have additionally analyzed the activity-surface area trend with respect to the pore origin (i.e. pores derived from nanoparticle agglomeration $v s$ template-derived pores) and the type of the template used to engineer pores, shown in Figure $2 b$ and $3 a$, respectively. Although a comparably smaller number of interparticle-derived mesoporous photocatalyst have been investigated, the results in Figure $2 \mathrm{~b}$ also indicate that the origin of the pores is not the major factor defining the non-linear relation of surface area with activity.

In contrast, we observed that the activity increase with surface area (Figure 3a) strongly depends on the type of porogen. For example, catalysts with pores that were engineered with hard templates showed a close to linear correlation in comparison with soft-templated samples. Hard-templating, when performed appropriately, can lead to well-defined pores with narrow pore size distribution. This could be an indication that narrow pore size distribution can be of benefit for the reactant and educts diffusion.
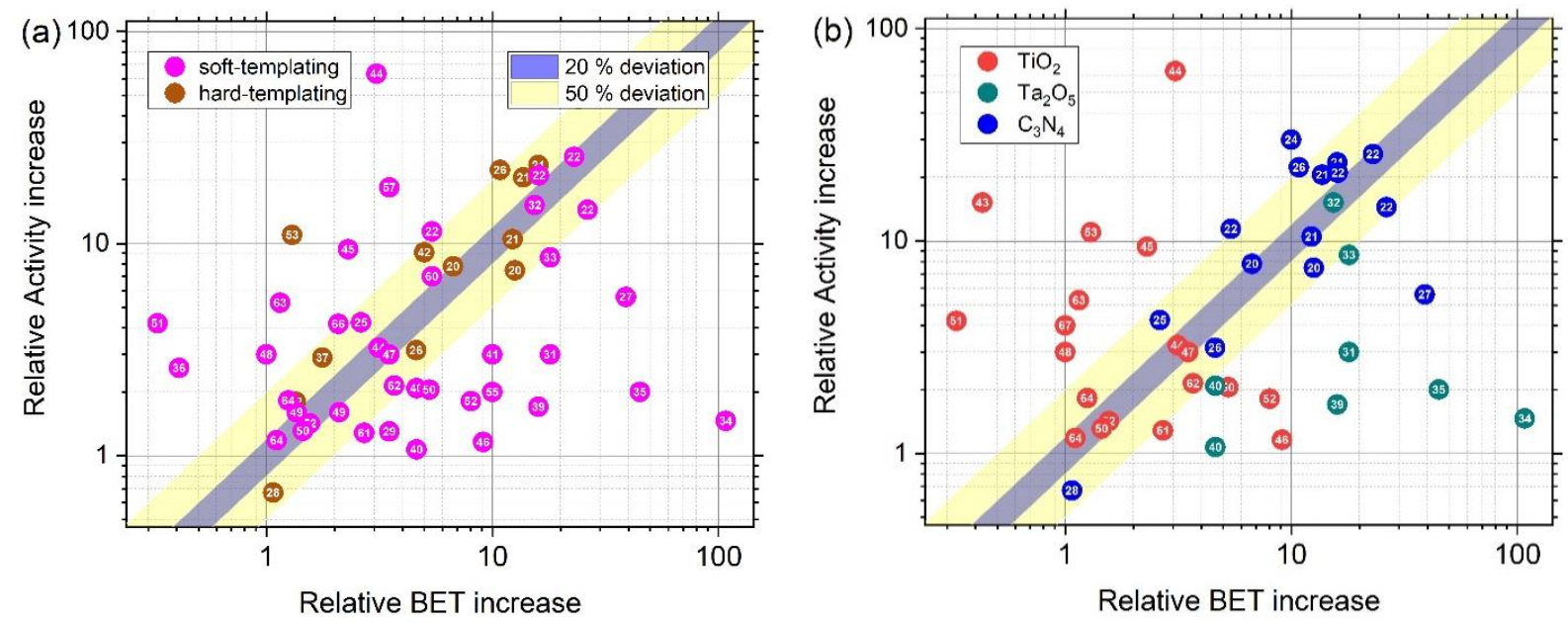

Figure 3 Correlation of increase in photocatalytic or photoelectrochemical activity with increase in BET surface area, both related to a non-porous reference as a function of (a) template nature; (b) material. Different colors represent different application. Colored areas represent deviation from the linear surfaceactivity correlation. Numbers inside the data points correspond to the reference number of the manuscript in the main reference list.

In another set of analyses, we extracted the data for different types of material (Figure 3b). It is very interesting to see that carbon nitride (or polyheptazine) generally seems to enable large surface area and activity increases: many studies on mesoporous carbon nitride show activity and surface area enlargements by a factor of 10 or more. Moreover, in many cases there is only a slight deviation from a linear correlation. This trend indicates 
that increasing surface area of carbon nitrides is a viable strategy to improve their photocatalytic performance.

In contrast, the results for both $\mathrm{TiO}_{2}$ and $\mathrm{Ta}_{2} \mathrm{O}_{5}$ are much more scattered and large deviations from the linear correlation can be observed. Interestingly, most of the $\mathrm{Ta}_{2} \mathrm{O}_{5}$ data points lie far off the linear relationship region: only one example with large ordered mesopores shows linear activity increase with surface area enlargement. ${ }^{34}$ Still, most of the data points for $\mathrm{Ta}_{2} \mathrm{O}_{5}$ are concentrated on the bottom right corner of the graph (Figure 3b). For example, some reports described $\mathrm{Ta}_{2} \mathrm{O}_{5}$ materials with up to 100 -times higher surface areas compared to a reference material, ${ }^{36}$ yet with only a minor activity increase. This suggests that the strategy for activity enhancement by increasing the surface area has not been successful in the case of $\mathrm{Ta}_{2} \mathrm{O}_{5}$ yet, as apparently different parameters govern its photocatalytic performance.

\subsection{What is the reason for this diverse behavior?}

Our data analysis and model have shed new light on the impact of prominent criteria, however, it remains a single parameter analysis and we also need to consider the combination of parameters. In fact, we could not find one study yet that varied exclusively one structural parameter. Referring again to the aforementioned $\mathrm{Ta}_{2} \mathrm{O}_{5}$ examples: both mesoporous samples were prepared by soft-templating, but still the resulting pore size characteristics differed strongly. Moreover, the study with the strongest deviation from linear behavior actually compared an amorphous mesoporous $\mathrm{Ta}_{2} \mathrm{O}_{5}$ with a bulk crystalline reference ${ }^{36}$ Consequently, differences in both, crystallinity and pore size distribution, have likely influenced the performance in addition to the surface area and porosity. This may explain the strongly scattered results and why it remains a challenge to identify the key parameter in designing mesoporous photo(electro)catalysts.

Another challenge in identifying the key parameters is the fact that many of them have a strong overlap. As such, when analyzing the impact of the sacrificial agent type on the activity-surface trend, we have found that $>95 \%$ of the reports in which trimethylamine was used as an electron donor have exclusively employed carbon nitride materials as photo(electro)catalysts (see Figure S1). This example illustrates that the existing 
literature does not provide any means to deconvolute these two factors and understand the effect of the sacrificial agent type and structure on photocatalytic performance that might be affected by pore diffusion in a complex way.

Finally, many of the analyzed reports used a reference material that was not fully characterized to correlate activity with surface area. For example, some reports used commercial samples without providing any analysis of phase composition or crystallinity or crystallite size; other works used different temperature treatments for the reference again without accounting for changes in phase composition and crystallinity. Sometime, no information on the reference has been provided at all.

A suitable reference could be prepared in the same way (e.g. technique, protocol) and post-treated under the same conditions (e.g. temperature) as the mesoporous material, yet without the porogen. Thus, an ideal reference would mimic the crystallite size, crystallinity, defect concentration and impurity level of the mesoporous sample, yet exhibit a lower surface area. It is clear that a reference needs to be adjusted for every respective material and application, and that its design constantly imposes a challenge in materials chemistry.

Our analysis revealed that, for whatever reasons, nearly $80 \%$ of the papers did not investigate suitable references (see the full list of references in the $\mathrm{SI}$ ). This reduces the database to the small, yet significant set of studies presented in Table 1 for further evaluation. However, we believe that our proposed model and methodology is very useful to interpret photocatalysis results of mesoporous photocatalysts with a new perspective. This attempt might provide a basis for future analysis given more and better-designed catalyst and reference systems being available. This analysis should also draw the attention of the readers to the need to evaluate literature results on mesoporous photocatalysts more carefully and critically in the future.

\subsection{Exemplary efforts and their takeaways}

As already mentioned, it is very difficult to control all material properties of a mesoporous photo(electro)catalyst at a time while synthetically varying only one. We have selected several reports in literature which, in our opinion, have put great effort into purposeful 
synthesis, analysis and control of mesoporous photocatalysts, or have evaluated and discussed the interplay between various parameters.

A number of research works aimed to relate photocatalytic activity with the BET surface area of the prepared photocatalysts. As such, Puangpetch et al. ${ }^{53}$ prepared a $\mathrm{SrTiO}_{3}$ powder with narrow-sized mesopores and compared it with a commercial powder that exhibited broad-sized interparticular pores, yet similar crystalline phase and crystallite size. The authors highlighted the importance of the small particle size of the mesoporous sample that was similar to the crystallite size unlike in the reference sample that consisted of significantly larger particles. Their $\mathrm{H}_{2}$ evolution tests performed with different sacrificial agents suggest that the values of specific surface area should not be considered as the global indicator for photocatalytic activity. Instead, parameters such as porosity, pore and wall size, uniformity and size of crystallites and particles as well as their interplay should to be given more attention.

In a similar way, Masolo et al. ${ }^{63}$ evaluated the role of various synthetic conditions on morphology, texture and phase content of non-ordered, yet mesoporous $\mathrm{TiO}_{2}$ powders were prepared via a soft-templating route. Their photoelectrochemical tests suggest that neither the pore volume nor specific surface area plays a key role in the performance. Instead, homogeneous intra- and inter-particles pore distribution has been mentioned as the most important parameter. In general, a uniform distribution leads to an optimized mass transfer and, as a consequence, to a higher activity.

To a similar conclusion came Kailasam et al., ${ }^{30}$ who synthesized mesoporous carbonnitride silica composites via combined sol-gel/thermal condensation route and critically discussed several effects that can influence the activity of mesoporous semiconductors. The measured increase in photocatalytic activity for water reduction was not linearly proportional to the increase of surface area of the materials due to many additional parameters, such as the different pore wall thicknesses. The tricky relationship between the size of the pores, the number and accessibility of surface active sites, the pore wall thickness (smaller pores yielded higher number of surface sites and thinner pore walls, which could yield numerous defects), and the corresponding activity was also mentioned by the authors as an important challenge for further studies. 
Tüysüz et al. ${ }^{40}$ also mentioned the influence of crystallinity, particle size, structure and morphology on the photocatalytic activity. They identified two important effects that gave rise to higher rates: improved crystallinity (that yields fewer recombination centers for charge trapping) and enlarged surface area of the photocatalyst (that yields more active sites for substrate adsorption and reaction). However, they acknowledged that high crystallinity often requires high temperature treatments which in turn increase particle size and decrease available surface area. A trade-off between these characteristics needs to be carefully considered.

Lin et al. ${ }^{31}$ provided another experimental evidence for this trend. The authors evaluated photocatalytic activity for water splitting over wormhole-like mesoporous $\mathrm{Nb}_{2} \mathrm{O}_{5}$ (see Figure 4a) and discussed the competition between the crystallinity and the surface area as a result of different heat treatments with regard to the photocatalytic activity. With higher calcination temperatures, the surface area of the materials was decreased while the crystal sizes increased. The mesoporous $\mathrm{Nb}_{2} \mathrm{O}_{5}$ calcined at $650^{\circ} \mathrm{C}$ showed a lower hydrogen production rate compared to the $450{ }^{\circ} \mathrm{C}$ calcined sample due to the larger surface area of the latter. However the specific surface photocatalytic activity was higher for the $650^{\circ} \mathrm{C}$ calcined catalyst as a result of higher degree of crystallinity. In another example Kuo et al..$^{57}$ prepared a series of mesoporous $\mathrm{Mn}_{2} \mathrm{O}_{3}$ via soft-templating, and investigated the dependence of the oxygen evolution activity on the porosity and crystallinity, in comparison to a non-porous commercial sample. The most crystalline commercial $\mathrm{Mn}_{2} \mathrm{O}_{3}$ showed the highest surface area normalized activity, indicating the crucial role of crystallinity. One the other hand, the maximum absolute activity was achieved with a mesoporous $\mathrm{Mn}_{2} \mathrm{O}_{3}$ sample exhibiting the highest surface area of 150 $\mathrm{m}^{2} \mathrm{~g}^{-1}$. These works demonstrate the complexity of mesoporous photo(electro)catalysis, which, depending on the choice of material and reaction, can render different structural parameters to become dominant and in fact limit catalytic performance.

There are only few reports, in which the authors have tried to prepare mesoporous photocatalysts with different pore size characteristics to elucidate whether the pore size actually matters. In one example, Weller et al. ${ }^{41}$ prepared a quaternary semiconductor, $\mathrm{CsTaWO}_{6}$, with various mesoporous morphologies. By optimizing synthesis conditions, 
they obtained samples with nearly identical crystallinity, yet altered pore morphology, and investigated the influence of pore size and pore size distribution on photocatalytic hydrogen production activity. Although the derived mesopores were not ordered, the authors showed that pores of 5-7 nm were too small (poorly accessible) to improve hydrogen evolution, even though the surface area was increased by 10 times. Only when the pore size was increased to up to $20 \mathrm{~nm}$, the increase in surface area (up to $78 \mathrm{~m}^{2} \mathrm{~g}$ $\left.{ }^{1}\right)$ resulted in doubled activity compared to the non-porous reference. Still, the activity increase did not go in line with the increase in surface area. Recently, Weller et al. presented ordered mesoporous $\mathrm{CsTaWO}_{6}$ with mesopores around $40 \mathrm{~nm}$ in size (Figure 4b). ${ }^{75}$ Comparing both mesoporous $\mathrm{CsTaWO}_{6}$, the ordered mesoporous material showed $300 \%$ increased activity in hydrogen production albeit reduced surface area. This shows that even with decreased surface area strong activity enhancements can be achieved when large mesopores for improved mass transport are established in semiconductor oxide materials.

In another contribution, Cherevan et al. ${ }^{34}$ reported on the synthesis of ordered mesoporous $\mathrm{Ta}_{2} \mathrm{O}_{5}$ with large pore sizes beyond $30 \mathrm{~nm}$ and narrow pore size distribution (Figure 4c). The authors showed that the 3D-interconnected gyroid mesopore architecture in the combination with sufficiently large pores can effectively eliminate diffusion limitations observed in previously reported mesoporous $\mathrm{Ta}_{2} \mathrm{O}_{5}$ with smaller or random pores. As a result, this mesoporous photocatalyst revealed a unique linear activity increase with respect to the surface area enlargement when compared to a powdered reference material prepared using similar conditions but without the porogen. These works show that pore size characteristics need to be considered since the presence of too small pores can trigger undesirable mass transport limitations.

Several works were dedicated to synthesis and activity evaluation of photo(electro)catalysts exhibiting various pore size distribution. For example, Pan et al.54 reported on the synthesis of mesoporous perovskite titanate spheres with tunable porosity characteristics. The authors managed to prepare hollow $\mathrm{SrTiO}_{3}$ spheres with hierarchical porosity (centered at 3 and $100 \mathrm{~nm}$ ) and solid $\mathrm{SrTiO}_{3}$ spheres with unimodal (centered at $20 \mathrm{~nm}$ ) mesopores, yet with comparable sphere size, specific surface area, absorption 
properties and crystalline phase. Interestingly, despite huge differences in the pore size distributions and the presence of small mesopores in the former sample, photocatalytic $\mathrm{O}_{2}$ evolution rates normalized per surface area yielded similar activity values (within 10\%). This result suggests that building photocatalysts with hierarchical pores can effectively address diffusion limitations inherent to small mesopores and render the catalyst matrix fully accessible for reactants and products.

Another example of hierarchical macro-mesoporous photocatalysts was reported by Zhou et al. ${ }^{59}$ This work compared PEC water splitting activity of $\mathrm{BiVO}_{4}$ films with dual porosity. They reported a striking difference in activity, when normalized to electrode specific surface area, which demonstrated the importance of the additional macropores in the final film. Again, the authors conclude that such hierarchically ordered macro-mesostructure (exemplary image in Fig. 4d) can increase the accessibility of the internal surface created by the small mesopores.

In a different contribution, Fang et al. ${ }^{69}$ photoreduced $\mathrm{CO}_{2}$ to $\mathrm{CH}_{4}$ using Pt-modified mesoporous $\mathrm{TiO}_{2}$ microspheres made of nanocrystals with similar anatase-to-rutile ratio compared to commercial P25. The spheres had also similar surface areas yet differed in their pore size distributions, with dual maxima at 2.3 and $12.4 \mathrm{~nm}$ for the sol-gel samples and a single peak centered at $\sim 3 \mathrm{~nm}$ for P25-derived microspheres. Due to the presence of large mesopores, the activity for $\mathrm{CO}_{2}$ reduction was increased by a factor of $\sim 5$. This result was attributed to the hierarchical porosity, being beneficial for fast mass transport and enhanced light harvesting. These works show the ability of the meso-macroporous systems to cope with inefficient mass transport kinetics intrinsic to mere mesopores making it a viable strategy to fully utilize benefits of mesoporous photo(electro)catalysts.

Only few reports have been trying to identify the advantage of 3D-interconnected ordered mesoporosity. Hossain et al. ${ }^{46}$ prepared mesoporous $\mathrm{TiO}_{2}$ photocatalysts with uniform hexagonal and with random wormhole-like pores (Figure 4e). Both materials appeared highly crystalline and exhibited similar crystallite/wall dimensions as well as comparable specific surface areas and pore sizes lying within the 3-7 $\mathrm{nm}$ range. Photocatalytic tests, however, revealed strong differences in activity towards $\mathrm{H}_{2}$ evolution reaction, with the ordered porous $\mathrm{TiO}_{2}$ outperforming the random porous $\mathrm{TiO}_{2}$ by the factor of at least 20 . 

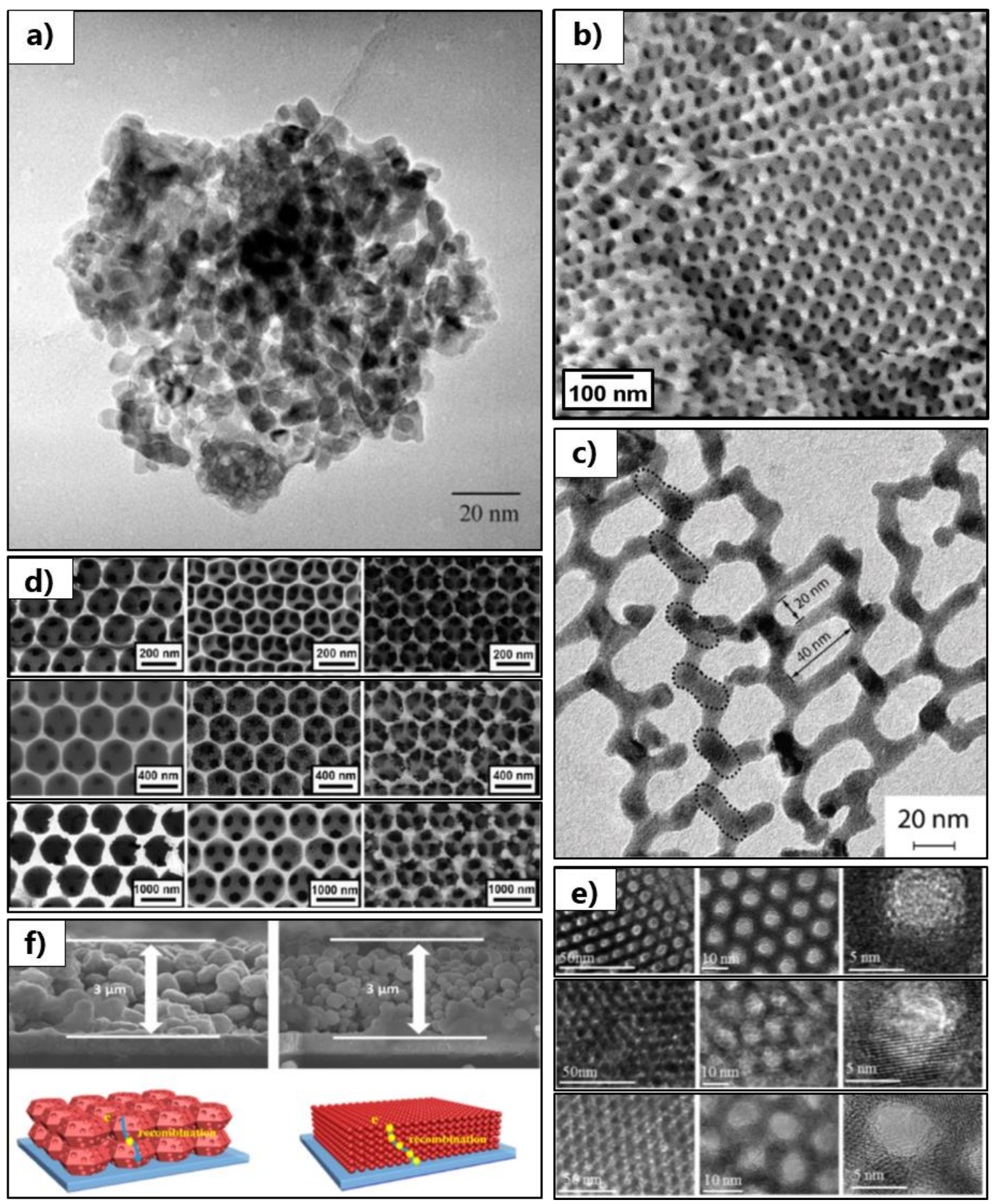

Figure 4 (a) TEM images of mesoporous $\mathrm{Nb}_{2} \mathrm{O}_{5}$ exhibiting wormhole-like porosity; reproduced with permission from ref. ${ }^{31}$, (C) 2011 Elsevier; (b) SEM image of mesoporous $\mathrm{CsTaWO}_{6}$ with ordered porous structure; reproduced with permission from ref. ${ }^{75}$, (c) 2018 The Royal Society of Chemistry (c) TEM image of mesoporous gyroid $\mathrm{Ta}_{2} \mathrm{O}_{5}$ fragment with large uniform pores; reproduced with permission from ref. ${ }^{34}(\mathrm{C}$ 2016 The Royal Society of Chemistry; (d) SEM images of ordered macroporous $\mathrm{BiVO}_{4}$ with dual porosity; reproduced with permission from ref. ${ }^{61}$, (c) 2013 Wiley-VCH (e) TEM images of mesoporous $\mathrm{TiO}_{2}$ with uniform hexagonal pores; reproduced with permission from ref. ${ }^{46}$, (c) 2015 Elsevier (f) SEM images of hematite films prepared from mesoporous and solid single crystals; reproduced with permission from ref. 62, () 2016 American Chemical Society. 
Similar trends have been derived from degradation experiments including methylene blue and 4-chlorphenol photooxidation. Importantly, the authors attributed such superior activity of the ordered mesoporous sample to (1) uniform channel size that results in uniform diffusion rates of the reactant and products throughout the material, (2) open and accessible porosity as well as (3) faster transfer of charge carriers within the crystalline walls due to the presence of three-dimensional interconnected $\mathrm{TiO}_{2}$ matrix.

Mesoporous photo(electro)catalysts have been also studied with regard to other parameters and characteristics of the porous matrix. As such, Wang et al. ${ }^{62}$ prepared $\alpha$ $\mathrm{Fe}_{2} \mathrm{O}_{3}$ photoelectrodes from solid single crystals and mesoporous single crystals (Figure 4f). The latter were synthesized using hydrothermal approach, adding $\mathrm{NaF}$ and/or colloidal silica to the mixture to alter the morphology. The maximum IPCE of $20.2 \%$ at $360 \mathrm{~nm}$ was achieved with highly porous hematite. The activity of the mesoporous electrode increased 7-fold compared to dense films (made of solid single crystals), while the surface area increased nearly identically, by a factor of 5.4 (up to $18 \mathrm{~m}^{2} \mathrm{~g}^{-1}$ ) and crystallinity was kept the same. The authors refer their increased activity to superior light absorption and enhanced charges separation by the mesoporous electrode. Unfortunately, the authors did not provide information on the surface area after all synthetic variations, thus a definite trend could not be observed.

\section{Challenges and Outlook}

For more than a decade, considerable research efforts have been devoted toward designing photocatalysts and photoelectrodes with large specific surface areas via nanostructuring or/and pore engineering. It is commonly accepted that a larger surface area is beneficial for photocatalytic activity as it provides a higher number of active sites. But it also increase the probability of surface recombination.

In their anxiety to increase the surface area, researchers have aimed at introducing small pores, i.e. micropores and mesopores below $5 \mathrm{~nm}$. Such small pores, however, can hinder direct access to the interior surface, thus considerably limiting the performance of a catalyst. Kinetic restrictions due to pore diffusion therefore constitute a major challenge that is increasingly gaining awareness in the research community. 
Understandably, there is a trade-off between too narrow and too large pore sizes, and the ideal pore dimensions and optimum surface areas have each to be identified for the various types of materials and applications. Moreover, we are not aware of any study that was able to tune the pore size of a semiconductor with narrow pore size distribution without altering any other material properties. This remains a major challenge in the field of mesoporous photocatalysts. However, as confirmed in this review, hierarchical pore systems may in fact provide a promising alternative to improve mass transport into the pores of photocatalysts or photoelectrodes.

For most semiconductor photocatalysts, co-catalyst loading (e.g. noble metals, metal complexes) is necessary to obtain a measurable reaction rate. This is especially true for multielectron transfer reactions like water splitting and $\mathrm{CO}_{2}$ reduction. However, a homogeneous deposition of co-catalysts also requires full accessibility of internal surface area. An early example for diffusion-limited photodeposition was provided for mesoporous CsTaWO6. We observed that the activity of $\mathrm{CsTaWO}_{6}$ with small mesopores did not improve with increasing amounts of Rh co-catalyst, in contrast to $\mathrm{CsTaWO}_{6}$ with large ordered mesopores, which showed a linear enhancement with Rh loading. 40,75 Further studies are necessary to address the distribution of co-catalysts inside mesopores, and its influence on photocatalytic activity.

Another challenge involves the crystallinity, i.e. crystallite size, phase composition and defect/impurity level, of the photocatalyst. These are material-dependent parameters that strongly influence the optimum surface area as well as charge transport and extraction characteristics. For example, depending on the material, the diffusion length of charge carriers is different, which in turn affect the optimum crystallite size for both, absorption and diffusion of charge carriers. Nevertheless, surface recombination must be minimized, thus there is always an optimum ratio between crystallite size and surface area for each material - another trade-off to consider. As revealed by our analysis, increasing the surface area can be a promising route towards improving the photocatalytic activity, but only in cases where surface recombination is negligible, e.g. for carbon nitrides, which showed the expected linear increase in activity with surface area. 
Surprisingly, we could not find any study yet that explicitly underlines the advantages of pore ordering over non-ordered porosity for photocatalysis. This is understandable, as it is indeed very challenging to prepare mesoporous semiconductor materials with identical pore size and pore size distribution, yet tunable pore structures (e.g. 2D hexagonal vs. 3D cubic vs. non-ordered). Indeed, this might even be the most challenging task for a materials scientist in the field of mesoporous photocatalysis. As revealed in our study, hard-templating constitutes the most suitable method to introduce pores with uniform pore size distribution, albeit it may be limited to a narrow pore size range (i.e. either very small or very large pores) and only few pore architectures (e.g. spherical, cylindrical). In contrast, soft-templating, while synthetically more challenging, may offer a more versatile range of pore dimensions and structures.

A new parameter that has barely been investigated so far involves the optical properties of ordered mesoporous photocatalysts, which can affect the light penetration depth and absorption efficiency as well as induce additional photonic effects. There have already been some indications that mesoporous and hierarchical pore structures can exhibit multiple photon absorption due to scattering of light inside the pores. This area of research is still in its infancy, yet expected to stimulate much interest in future developments.

Finally, we would like to emphasize that many additional parameters and their effects on the photocatalytic performance of mesoporous catalysts should be investigated in the future. For example, not much is known about adsorption and diffusion of sacrificial agents within a mesopore system and their imminent influence on the photocatalytic activity. The same is valid for e.g. exposed surface facets inside the pores of porous photocatalysts. More comprehensive and focused studies are required to reveal these trends.

This review documents that a great amount of research in photocatalysis in the last few years has been directed at enhancing activity and efficiency of photocatalysts through mesostructuring and pore engineering. The database and methodology that we provide here can serve as a starting point for the community to extract key information on mesoporous photocatalysts, put results into context of existing data and better compare the performances of various catalytic systems. We believe it will stimulate the 
implementation of new concepts, such as optical/photonic effects in ordered pore architectures to further benefit the designing of a new generation of photocatalysts and photoelectrodes.

\section{Acknowledgement}

T.W. and R.M. gratefully acknowledge funding in the Emmy-Noether programm (MA 5392/3-1) of the German Research Foundation (DFG). D.E. also acknowledges funding by the DFG (ED 221/3-1).

\section{Supporting Information}

Relative activity increase depending on the used sacrificial agent, full list of references. Supporting Information is available from here.

\section{References}

1. Lewis, N. S.; Nocera, D. G., Powering the planet: Chemical challenges in solar energy utilization. Proc. Natl. Acad. Sci. U. S. A. 2007, 104 (50), 20142-20142.

2. Kudo, A.; Miseki, Y., Heterogeneous photocatalyst materials for water splitting. Chem. Soc. Rev. 2009, 38 (1), 253-278.

3. Habisreutinger, S. N.; Schmidt-Mende, L.; Stolarczyk, J. K., Photocatalytic Reduction of CO2 on TiO2 and Other Semiconductors. Angew. Chem.-Int. Edit. 2013, 52 (29), 7372-7408.

4. Daugulis, A. J., Two-phase partitioning bioreactors: a new technology platform for destroying xenobiotics. Trends Biotechnol. 2001, 19 (11), 457-462.

5. Marschall, R.; Wang, L. Z., Non-metal doping of transition metal oxides for visible-light photocatalysis. Catal. Today 2014, 225, 111-135.

6. Takata, T.; Domen, K., Defect Engineering of Photocatalysts by Doping of Aliovalent Metal Cations for Efficient Water Splitting. J. Phys. Chem. C 2009, 113 (45), 19386-19388.

7. $\quad$ Nolan, M.; Iwaszuk, A.; Lucid, A. K.; Carey, J. J.; Fronzi, M., Design of Novel Visible Light Active Photocatalyst Materials: Surface Modified TiO2. Adv. Mater. 2016, 28 (27), 5425-5446.

8. Li, H. J.; Zhou, Y.; Tu, W. G.; Ye, J. H.; Zou, Z. G., State-of-the-Art Progress in Diverse Heterostructured Photocatalysts toward Promoting Photocatalytic Performance. Adv. Funct. Mater. 2015, 25 (7), 998-1013.

9. $\quad$ Maeda, K.; Teramura, K.; Lu, D. L.; Saito, N.; Inoue, Y.; Domen, K., Noble-metal/Cr2O3 core/shell nanoparticles as a cocatalyst for photocatalytic overall water splitting. Angew. Chem.Int. Edit. 2006, 45 (46), 7806-7809.

10. Zhang, Y. H.; Zhang, N.; Tang, Z. R.; Xu, Y. J., Graphene Transforms Wide Band Gap ZnS to a Visible Light Photocatalyst. The New Role of Graphene as a Macromolecular Photosensitizer. ACS Nano 2012, 6 (11), 9777-9789.

11. Cherevan, A. S.; Gebhardt, P.; Shearer, C. J.; Matsukawa, M.; Domen, K.; Eder, D., Interface engineering in nanocarbon-Ta2O5 hybrid photocatalysts. Energy Environ. Sci. 2014, 7 (2), 791-796.

12. Osterloh, F. E., Nanoscale Effects in Water Splitting Photocatalysis. In Solar Energy for Fuels, Tuysuz, H.; Chan, C. K., Eds. Springer Int Publishing Ag: Cham, 2016; Vol. 371, pp 105142. 
13. Osterloh, F. E., Photocatalysis versus Photosynthesis: A Sensitivity Analysis of Devices for Solar Energy Conversion and Chemical Transformations. Acs Energy Letters 2017, 2 (2), 445453.

14. Schwertmann, L.; Wark, M.; Marschall, R., Sol-gel synthesis of defect-pyrochlore structured CsTaWO6 and the tribochemical influences on photocatalytic activity. RSC Adv. 2013, 3 (41), 18908-18915.

15. Gadiyar, C.; Loiudice, A.; Buonsanti, R., Colloidal nanocrystals for photoelectrochemical and photocatalytic water splitting. J. Phys. D-Appl. Phys. 2017, 50 (7), 22.

16. Gu, D.; Schuth, F., Synthesis of non-siliceous mesoporous oxides. Chem. Soc. Rev. 2014, 43 (1), 313-344.

17. Linares, N.; Silvestre-Albero, A. M.; Serrano, E.; Silvestre-Albero, J.; Garcia-Martinez, J., Mesoporous materials for clean energy technologies. Chem. Soc. Rev. 2014, 43 (22), 7681-7717.

18. Yang, X. Y.; Chen, L. H.; Li, Y.; Rooke, J. C.; Sanchez, C.; Su, B. L., Hierarchically porous materials: synthesis strategies and structure design. Chem. Soc. Rev. 2017, 46 (2), 481-558.

19. Sick, T.; Hufnagel, A. G.; Kampmann, J.; Kondofersky, I.; Calik, M.; Rotter, J. M.; Evans, A.; Doblinger, M.; Herbert, S.; Peters, K.; Bohm, D.; Knochel, P.; Medina, D. D.; FattakhovaRohlfing, D.; Bein, T., Oriented Films of Conjugated 2D Covalent Organic Frameworks as Photocathodes for Water Splitting. J. Am. Chem. Soc. 2018, 140 (6), 2085-2092.

20. Li, H.; Sun, Y.; Yuan, Z.-Y.; Zhu, Y.-P.; Ma, T.-Y., Titanium Phosphonate Based MetalOrganic Frameworks with Hierarchical Porosity for Enhanced Photocatalytic Hydrogen Evolution. Angewandte Chemie International Edition 2018, 57 (12), 3222-3227.

21. Lakhi, K. S.; Park, D. H.; Al-Bahily, K.; Cha, W.; Viswanathan, B.; Choy, J. H.; Vinu, A., Mesoporous carbon nitrides: synthesis, functionalization, and applications. Chem. Soc. Rev. 2017, 46 (1), 72-101.

22. Wang, X. C.; Maeda, K.; Chen, X. F.; Takanabe, K.; Domen, K.; Hou, Y. D.; Fu, X. Z.; Antonietti, M., Polymer Semiconductors for Artificial Photosynthesis: Hydrogen Evolution by Mesoporous Graphitic Carbon Nitride with Visible Light. J. Am. Chem. Soc. 2009, 131 (5), 1680+ .

23. Schroder, M.; Kailasam, K.; Rudi, S.; Richter, M.; Thomas, A.; Schomacker, R.; Schwarze, M., Impact of the reaction conditions on the photocatalytic reduction of water on mesoporous polymeric carbon nitride under sunlight irradiation. Int. J. Hydrog. Energy 2014, 39 (19), 1010810120.

24. Hollmann, D.; Karnahl, M.; Tschierlei, S.; Kailasam, K.; Schneider, M.; Radnik, J.; Grabow, K.; Bentrup, U.; Junge, H.; Beller, M.; Lochbrunner, S.; Thomas, A.; Bruckner, A., StructureActivity Relationships in Bulk Polymeric and Sol-Gel-Derived Carbon Nitrides during Photocatalytic Hydrogen Production. Chem. Mat. 2014, 26 (4), 1727-1733.

25. Chen, X. F.; Jun, Y. S.; Takanabe, K.; Maeda, K.; Domen, K.; Fu, X. Z.; Antonietti, M.; Wang, X. C., Ordered Mesoporous SBA-15 Type Graphitic Carbon Nitride: A Semiconductor Host Structure for Photocatalytic Hydrogen Evolution with Visible Light. Chem. Mat. 2009, 21 (18), 4093-4095.

26. Han, Q.; Wang, B.; Zhao, Y.; Hu, C. G.; Qu, L. T., A Graphitic-C3N4 "Seaweed" Architecture for Enhanced Hydrogen Evolution. Angew. Chem.-Int. Edit. 2015, 54 (39), 1143311437.

27. Shi, L.; Liang, L.; Wang, F. X.; Liu, M. S.; Chen, K. L.; Sun, K. N.; Zhang, N. Q.; Sun, J. M., Higher Yield Urea-Derived Polymeric Graphitic Carbon Nitride with Mesoporous Structure and Superior Visible-Light-Responsive Activity. ACS Sustain. Chem. Eng. 2015, 3 (12), 3412-3419.

28. Hong, J. D.; Xia, X. Y.; Wang, Y. S.; Xu, R., Mesoporous carbon nitride with in situ sulfur doping for enhanced photocatalytic hydrogen evolution from water under visible light. J. Mater. Chem. 2012, 22 (30), 15006-15012. 
29. Zhu, Y. P.; Ren, T. Z.; Yuana, Z. Y., Mesoporous Phosphorus-Doped g-C3N4 Nanostructured Flowers with Superior Photocatalytic Hydrogen Evolution Performance. ACS Appl. Mater. Interfaces 2015, 7 (30), 16850-16856.

30. Kailasam, K.; Epping, J. D.; Thomas, A.; Losse, S.; Junge, H., Mesoporous carbon nitridesilica composites by a combined sol-gel/thermal condensation approach and their application as photocatalysts. Energy Environ. Sci. 2011, 4 (11), 4668-4674.

31. Lin, H. Y.; Yang, H. C.; Wang, W. L., Synthesis of mesoporous Nb2O5 photocatalysts with $\mathrm{Pt}, \mathrm{Au}, \mathrm{Cu}$ and $\mathrm{NiO}$ cocatalyst for water splitting. Catal. Today 2011, 174 (1), 106-113.

32. Lin, H. Y.; Huang, H. C.; Wang, W. L., Preparation of mesoporous In-Nb mixed oxides and its application in photocatalytic water splitting for hydrogen production. Microporous Mesoporous Mat. 2008, $115(3), 568-575$.

33. Guo, L. M.; Hagiwara, H.; Ida, S.; Daio, T.; Ishihara, T., One-Pot Soft-Templating Method to Synthesize Crystalline Mesoporous Tantalum Oxide and Its Photocatalytic Activity for Overall Water Splitting. ACS Appl. Mater. Interfaces 2013, 5 (21), 11080-11086.

34. Cherevan, A. S.; Robbins, S.; Dieterle, D.; Gebhardt, P.; Wiesner, U.; Eder, D., Ordered gyroidal tantalum oxide photocatalysts: eliminating diffusion limitations and tuning surface barriers. Nanoscale 2016, 8 (37), 16694-16701.

35. Noda, Y.; Lee, B.; Domen, K.; Kondo, J. N., Synthesis of Crystallized Mesoporous Tantalum Oxide and Its Photocatalytic Activity for Overall Water Splitting under Ultraviolet Light Irradiation. Chem. Mat. 2008, 20 (16), 5361-5367.

36. Grewe, T.; Tuysuz, H., Alkali metals incorporated ordered mesoporous tantalum oxide with enhanced photocatalytic activity for water splitting. J. Mater. Chem. A 2016, 4 (8), 3007-3017. 37. Zhou, C.; Shang, L.; Yu, H. J.; Bian, T.; Wu, L. Z.; Tung, C. H.; Zhang, T. R., Mesoporous plasmonic Au-loaded Ta2O5 nanocomposites for efficient visible light photocatalysis. Catal. Today 2014, 225, 158-163.

38. Guo, L. M.; Ida, S.; Daio, T.; Hagiwara, H.; Ishihara, T., In situ carbonization of a softtemplate to directly synthesize crystalline mesoporous metal oxides with high surface areas. New J. Chem. 2014, 38 (12), 5846-5855.

39. Yokoi, T.; Sakuma, J.; Maeda, K.; Domen, K.; Tatsumi, T.; Kondo, J. N., Preparation of a colloidal array of $\mathrm{NaTaO} 3$ nanoparticles via a confined space synthesis route and its photocatalytic application. Phys. Chem. Chem. Phys. 2011, 13 (7), 2563-2570.

40. Tuysuz, H.; Chan, C. K., Preparation of amorphous and nanocrystalline sodium tantalum oxide photocatalysts with porous matrix structure for overall water splitting. Nano Energy 2013, 2 (1), 116-123.

41. Weller, T.; Sann, J.; Marschall, R., Pore Structure Controlling the Activity of Mesoporous Crystalline CsTaWO6 for Photocatalytic Hydrogen Generation. Adv. Energy Mater. 2016, 6 (16), 9.

42. Suzuki, T. M.; Nakamura, T.; Saeki, S.; Matsuoka, Y.; Tanaka, H.; Yano, K.; Kajino, T.; Morikawa, T., Visible light-sensitive mesoporous $\mathrm{N}$-doped Ta2O5 spheres: synthesis and photocatalytic activity for hydrogen evolution and CO2 reduction. J. Mater. Chem. 2012, 22 (47), 24584-24590.

43. Hisatomi, T.; Otani, M.; Nakajima, K.; Teramura, K.; Kako, Y.; Lu, D. L.; Takata, T.; Kondo, J. N.; Domen, K., Preparation of Crystallized Mesoporous Ta3N5 Assisted by Chemical Vapor Deposition of Tetramethyl Orthosilicate. Chem. Mat. 2010, 22 (13), 3854-3861.

44. Yuliati, L.; Yang, J. H.; Wang, X. C.; Maeda, K.; Takata, T.; Antonietti, M.; Domen, K., Highly active tantalum(v) nitride nanoparticles prepared from a mesoporous carbon nitride template for photocatalytic hydrogen evolution under visible light irradiation. J. Mater. Chem. 2010, 20 (21), 4295-4298.

45. Lakshminarasimhan, N.; Bae, E.; Choi, W., Enhanced photocatalytic production of H-2 on mesoporous $\mathrm{TiO} 2$ prepared by template-free method: Role of interparticle charge transfer. J. Phys. Chem. C 2007, 111 (42), 15244-15250. 
46. Hossain, M. K.; Akhtar, U. S.; Koirala, A. R.; Hwang, I. C.; Yoon, K. B., Steam-assisted synthesis of uniformly mesoporous anatase and its remarkably superior photocatalytic activities. Catal. Today 2015, 243, 228-234.

47. Moya, A.; Cherevan, A.; Marchesan, S.; Gebhardt, P.; Prato, M.; Eder, D.; Vilatela, J. J., Oxygen vacancies and interfaces enhancing photocatalytic hydrogen production in mesoporous CNT/TiO2 hybrids. Appl. Catal. B-Environ. 2015, 179, 574-582.

48. Yi, H. B.; Peng, T. Y.; Ke, D. N.; Ke, D.; Zan, L.; Yan, C. H., Photocatalytic H-2 production from methanol aqueous solution over titania nanoparticles with mesostructures. Int. J. Hydrog. Energy 2008, 33 (2), 672-678.

49. Kandiel, T. A.; Ismail, A. A.; Bahnemann, D. W., Mesoporous TiO2 nanostructures: a route to minimize $\mathrm{Pt}$ loading on titania photocatalysts for hydrogen production. Phys. Chem. Chem. Phys. 2011, 13 (45), 20155-20161.

50. Liu, S. H.; Syu, H. R., One-step fabrication of N-doped mesoporous $\mathrm{TiO} 2$ nanoparticles by self-assembly for photocatalytic water splitting under visible light. Appl. Energy 2012, 100, 148154.

51. Sang, L. X.; Dai, H. X.; Sun, J. H.; Xu, L. X.; Wang, F.; Ma, C. F., Fabrication of the hydrogen-evolving photocatalyst with mesoporous structure. Int. J. Hydrog. Energy 2010, 35 (13), 7098-7103.

52. $\quad$ Pany, S.; Parida, K. M., Sulfate-Anchored Hierarchical Meso-Macroporous N-doped TiO2: A Novel Photocatalyst for Visible Light H-2 Evolution. ACS Sustain. Chem. Eng. 2014, 2 (6), 14291438.

53. Puangpetch, T.; Sreethawong, T.; Yoshikawa, S.; Chavadej, S., Hydrogen production from photocatalytic water splitting over mesoporous-assembled SrTiO3 nanocrystal-based photocatalysts. J. Mol. Catal. A-Chem. 2009, 312 (1-2), 97-106.

54. Pan, J. H.; Shen, C.; Ivanova, I.; Zhou, N.; Wang, X. Z.; Tan, W. C.; Xu, Q. H.; Bahnemann, D. W.; Wang, Q., Self-Template Synthesis of Porous Perovskite Titanate Solid and Hollow Submicrospheres for Photocatalytic Oxygen Evolution and Mesoscopic Solar Cells. ACS Appl. Mater. Interfaces 2015, 7 (27), 14859-14869.

55. Yu, K.; Zhang, C. X.; Chang, Y.; Feng, Y. J.; Yang, Z. Q.; Yang, T.; Lou, L. L.; Liu, S. X., Novel three-dimensionally ordered macroporous SrTiO3 photocatalysts with remarkably enhanced hydrogen production performance. Appl. Catal. B-Environ. 2017, 200, 514-520.

56. Sathish, A.; Viswanath, R. P., Photocatalytic generation of hydrogen over mesoporous CdS nanoparticle: Effect of particle size, noble metal and support. Catal. Today 2007, 129 (3-4), 421-427.

57. Kuo, C. H.; Mosa, I. M.; Poyraz, A. S.; Biswas, S.; E-Sawy, A. M.; Song, W. Q.; Luo, Z.; Chen, S. Y.; Rusling, J. F.; He, J.; Suib, S. L., Robust Mesoporous Manganese Oxide Catalysts for Water Oxidation. ACS Catal. 2015, 5 (3), 1693-1699.

58. Liu, B.; Kuo, C. H.; Chen, J. J.; Luo, Z.; Thanneeru, S.; Li, W. K.; Song, W. Q.; Biswas, S.; Suib, S. L.; He, J., Ligand-Assisted Co-Assembly Approach toward Mesoporous Hybrid Catalysts of Transition-Metal Oxides and Noble Metals: Photochemical Water Splitting. Angew. Chem.-Int. Edit. 2015, 54 (31), 9061-9065.

59. Jang, J. S.; Yu, C. J.; Choi, S. H.; Ji, S. M.; Kim, E. S.; Lee, J. S., Topotactic synthesis of mesoporous $\mathrm{ZnS}$ and $\mathrm{ZnO}$ nanoplates and their photocatalytic activity. J. Catal. 2008, 254 (1), 144-155.

60. Zhang, L. W.; Baumanis, C.; Robben, L.; Kandiel, T.; Bahnemann, D., Bi2WO6 Inverse

Opals: Facile Fabrication and Efficient Visible-Light-Driven Photocatalytic and Photoelectrochemical Water-Splitting Activity. Small 2011, 7 (19), 2714-2720.

61. Zhou, M.; Wu, H. B.; Bao, J.; Liang, L.; Lou, X. W.; Xie, Y., Ordered Macroporous BiVO4 Architectures with Controllable Dual Porosity for Efficient Solar Water Splitting. Angew. Chem.Int. Edit. 2013, 52 (33), 8579-8583. 
62. Wang, C. W.; Yang, S.; Fang, W. Q.; Liu, P. R.; Zhao, H. J.; Yang, H. G., Engineered Hematite Mesoporous Single Crystals Drive Drastic Enhancement in Solar Water Splitting. Nano Lett. 2016, 16 (1), 427-433.

63. Masolo, E.; Meloni, M.; Garroni, S.; Mulas, G.; Enzo, S.; Baro, M. D.; Rossinyol, E.; Rzeszutek, A.; Herrmann-Geppert, I.; Pilo, M., Mesoporous Titania Powders: The Role of Precursors, Ligand Addition and Calcination Rate on Their Morphology, Crystalline Structure and Photocatalytic Activity. Nanomaterials 2014, 4 (3), 583-598.

64. Kim, K.; Thiyagarajan, P.; Ahn, H. J.; Kim, S. I.; Jang, J. H., Optimization for visible light photocatalytic water splitting: gold-coated and surface-textured TiO2 inverse opal nano-networks. Nanoscale 2013, 5 (14), 6254-6260.

65. Zhang, R. Y.; Shen, D. K.; Xu, M.; Feng, D.; Li, W.; Zheng, G. F.; Che, R. C.; Elzatahry, A. A.; Zhao, D. Y., Ordered Macro-/Mesoporous Anatase Films with High Thermal Stability and Crystallinity for Photoelectrocatalytic Water-Splitting. Adv. Energy Mater. 2014, 4 (8), 7.

66. Hidalgo, D.; Messina, R.; Sacco, A.; Manfredi, D.; Vankova, S.; Garrone, E.; Saracco, G.; Hernandez, S., Thick mesoporous TiO2 films through a sol gel method involving a non-ionic surfactant: Characterization and enhanced performance for water photo-electrolysis. Int. J. Hydrog. Energy 2014, 39 (36), 21512-21522.

67. DeSario, P. A.; Pietron, J. J.; DeVantier, D. E.; Brintlinger, T. H.; Stroud, R. M.; Rolison, D. R., Plasmonic enhancement of visible-light water splitting with Au-TiO2 composite aerogels. Nanoscale 2013, 5 (17), 8073-8083.

68. Park, H. A.; Choi, J. H.; Choi, K. M.; Lee, D. K.; Kang, J. K., Highly porous gallium oxide with a high $\mathrm{CO} 2$ affinity for the photocatalytic conversion of carbon dioxide into methane. J. Mater. Chem. 2012, 22 (12), 5304-5307.

69. Fang, B. Z.; Bonakdarpour, A.; Reilly, K.; Xing, Y. L.; Taghipour, F.; Wilkinson, D. P., Large-Scale Synthesis of TiO2 Microspheres with Hierarchical Nanostructure for Highly Efficient Photodriven Reduction of CO2 to CH4. ACS Appl. Mater. Interfaces 2014, 6 (17), 15488-15498.

70. Yan, S. C.; Ouyang, S. X.; Gao, J.; Yang, M.; Feng, J. Y.; Fan, X. X.; Wan, L. J.; Li, Z. S.; Ye, J. H.; Zhou, Y.; Zou, Z. G., A Room-Temperature Reactive-Template Route to Mesoporous ZnGa2O4 with Improved Photocatalytic Activity in Reduction of CO2. Angew. Chem.-Int. Edit. 2010, 49 (36), 6400-6404.

71. Osterloh, F. E., Inorganic nanostructures for photoelectrochemical and photocatalytic water splitting. Chem. Soc. Rev. 2013, 42 (6), 2294-2320.

72. Nicolai, A.; Monti, J.; Daniels, C.; Meunier, V., Electrolyte Diffusion in Gyroidal Nanoporous Carbon. J. Phys. Chem. C 2015, 119 (5), 2896-2903.

73. Li, L.; Krissanasaeranee, M.; Pattinson, S. W.; Stefik, M.; Wiesner, U.; Steiner, U.; Eder, D., Enhanced photocatalytic properties in well-ordered mesoporous WO3. Chemical Communications 2010, 46 (40), 7620-7622.

74. Melchers, S.; Uesbeck, T.; Winter, O.; Eckert, H.; Eder, D., Effect of Aluminum Ion Incorporation on the Bioactivity and Structure in Mesoporous Bioactive Glasses. Chem. Mat. 2016, 28 (10), 3254-3264.

75. Weller, T.; Deilmann, L.; Timm, J.; Dorr, T. S.; Beaucage, P. A.; Cherevan, A. S.; Wiesner, U. B.; Eder, D.; Marschall, R., A crystalline and 3D periodically ordered mesoporous quaternary semiconductor for photocatalytic hydrogen generation. Nanoscale 2018, 10 (7), 3225-3234. 